\title{
BACTERIOLOGICAL PROFILE AND ANTIBIOTIC SUSCEPTIBILITY PATTERN OF NEONATAL SEPTICAEMIA
}

\author{
Shilpy Singh ${ }^{1}$, Seema Bankar ${ }^{2}$ \\ ${ }_{13}^{\text {rd }}$ Year Post Graduate Student, Department of Microbiology, D. Y. Patil Medical College, Kolhapur, Maharashtra. \\ ${ }^{2}$ Associate Professor, Department of Microbiology, D. Y. Patil Medical College, Kolhapur, Maharashtra.
}

\begin{abstract}
Neonatal sepsis is one of the most common causes of neonatal mortality and morbidity, particularly in the developing countries. Appropriate clinical diagnosis and empirical treatment is crucial as pathogens causing sepsis and their antibiotic susceptibility pattern varies in different settings. The objective of this study was to determine the causative bacteria and pattern of susceptibility to antibiotics in NICU, which in turn may help in implementation of empirical therapy.
\end{abstract}

\section{MATERIALS AND METHOD}

A total of 100 blood samples were screened for sepsis in newborns less than 28 days old in this prospective study. The blood cultures of suspected cases were detected by using BACTEC blood culture systems and antibiotic susceptibility testing was done by using Kirby-Bauer disk diffusion method. This prospective observational study was conducted in the Department of Microbiology and Level III NICU in the Department of Paediatrics of D. Y. Patil Hospital and Research Centre during the period of two years 2013 to 2015 .

\section{RESULTS}

In this study out of 100 neonates 38 (38\%) showed sepsis and 62 (62\%) showed no sepsis. Most common organisms responsible for the sepsis were CONS followed by Klebsiella pneumonia and Acinetobacter sp. Gram negative organisms were $100 \%$ sensitive to Colistin, Imipenem and Meropenem while Gram positive organisms were 100\% sensitive to Azithromycin, Linezolid and Vancomycin.

\section{CONCLUSION}

The diagnostic capabilities of blood culture systems have improved over the last decade with the advent of automated continuous blood culture monitoring systems. BACTEC is a sensitive method and lead to earlier detection of bacterial growth.

\section{KEYWORDS}

Neonatal Sepsis, BACTEC Blood Culture Systems, Kirby-Bauer Disk Diffusion Method.

HOW TO CITE THIS ARTICLE: Singh S, Bankar S. Bacteriological profile and antibiotic susceptibility pattern of neonatal septicaemia. J. Evolution Med. Dent. Sci. 2016;5(51):3323-3327, DOI: 10.14260/jemds/2016/769

\section{INTRODUCTION}

Out of the one hundred and thirty million babies born every year worldwide, about four million die in the first four weeks of life - the neonatal period. ${ }^{1}$ The World Health Organization (WHO) estimates that, worldwide, approximately five million neonates die each year and that $98 \%$ of these deaths occur in developing countries. ${ }^{2}$

Neonatal mortality rate per 1000 live births varies from 5 in developed countries to 53 in the least developed countries. Neonatal infections are estimated to cost 1.6 million annual deaths or $40 \%$ of all neonatal deaths in developing countries. ${ }^{3}$ The incidence of Neonatal sepsis according to data from National Neonatal Perinatal Database (NNPD, 2002-03) is 30 per 1000 live births. Across India sepsis is found to be one of the commonest causes of neonatal mortality contributing to $19 \%$ of all neonatal deaths. ${ }^{4}$

Financial or Other, Competing Interest: None.

Submission 06-05-2016, Peer Review 03-06-2016,

Acceptance 09-06-2016, Published 27-06-2016.

Corresponding Author:

Dr. Shilpy Singh,

Flat No. 4C, Block-B,

Bhaskar Complex, Morhabadi,

Ranchi- 834008,

Jharkhand.

E-mail: shilpy.sd@gmail.com

DOI: $10.14260 /$ jemds/2016/769
Bacterial sepsis in the neonate is a clinical syndrome characterized by systemic signs of infection accompanied by bacteraemia in the first month of life. It encompasses systemic infections of the new born including septicaemia, meningitis, pneumonia, arthritis, osteomyelitis and urinary tract infections of the new born. Neonatal sepsis can be divided into two main classes depending on the onset of symptoms related to sepsis. Early onset sepsis usually presents within $72 \mathrm{hrs}$. of life. ${ }^{5}$ Source of infection is generally the maternal genital tract. It often manifests as pneumonia causing acute respiratory distress.

Late onset sepsis usually presents after 72 hours of birth. The source of infection is either nosocomial or community acquired. Neonate usually presents with septicaemia, pneumonia or meningitis. ${ }^{5}$

Various Factors that Predispose. 5 to an Increased Risk of Nosocomial Sepsis Include

- NICU admission.

- Invasive procedures.

- Parenteral fluid therapy.

- Low birth weight and prematurity.

- Ventilation and use of stock solution.

The early and efficient diagnosis of neonatal bacterial sepsis remains a difficult task. 
Blood cultures are the gold standard in the diagnosis of neonatal sepsis, but suffer from the disadvantages of low sensitivity and reporting delay of 24-72 h. The diagnostic capabilities of blood culture systems have improved over the last decade with the advent of automated continuous blood culture monitoring systems. ${ }^{5}$ The fact that the blind subculturing procedure of conventional methods has not been applied in the automated systems minimizes the risks of contamination. Also, the most important advantage of this system is the time it gains for treatment due to the rapid isolation, especially for slow-growing management of septicaemia in children, study of bacteriological profile along with the antimicrobial sensitivity pattern plays a great role.

\section{METHODOLOGY}

The present study was conducted in the Department of Microbiology at a Tertiary Health Care Centre at Kolhapur.

Hundred neonates born with risk factors of septicaemia were studied.

\section{Collection of Samples}

1. Samples were collected with all aseptic precautions.

2. Blood sample: Sample was collected prior to administration of any antibiotic therapy.

3. To reduce the chances of introducing skin contaminants, the venepuncture site was prepared as follows. 6

\section{METHODS}

1. Blood was collected with all aseptic precautions. Sterile gloves were worn to conduct the procedure.

2. The vein chosen to be drawn by touching the skin before it has been disinfected.

3. Using $70 \%$ alcohol, the skin over the venepuncture site was cleansed in a circle approximately $5 \mathrm{~cm}$ in diameter by rubbing vigorously and was allowed to air dry.

4. Starting in the centre of the circle, $2 \%$ tincture of iodine (or povidone-iodine) was applied in widening circles beginning from the centre to the periphery until the entire circle was saturated with iodine. The iodine was allowed to air dry on the skin for at least 1 minute.

5. If the site had to be touched after the preparation, the gloved fingers were disinfected in identical fashion.

6. Needle was inserted into the vein and blood was withdrawn.

7. After the needle was removed, the site was cleansed with $70 \%$ alcohol.

8. 2 to $5 \mathrm{~mL}$ of blood was drawn.

9. Blood drawn for culture was inoculated into the BacT/ALERT PF plus disposable Culture Vials. ${ }^{7}$

\section{LABORATORY PROCEDURES}

\section{Processing of Blood Sample}

After the blood was drawn with aseptic precautions, out of $5 \mathrm{~mL}$,

- $2 \mathrm{~mL}$ was directly added to the BacT/ALERT PF plus disposable Culture Vials.

- $1 \mathrm{~mL}$ was collected in plain tube for CRP estimation

- Remaining sample was collected in EDTA (Ethylene diamine tetra-acetic acid) tube used to estimate microESR and sent for estimation of total leukocyte count and absolute neutrophil count.

\section{METHODOLOGY}

Hundred neonates born with risk factors of septicaemia were studied. After recruitment 2-3 mL venous blood sample was collected within six hours of birth using aseptic technique; 2 $\mathrm{mL}$ of which was directly transferred into the BacT/ALERT PF plus disposable Culture Vials containing enriched broth (Soybean-Casein Digest broth with resins) and incubated in BacT/ALERT blood culture instrument at $35.5^{\circ} \mathrm{C} \pm 1.5^{\circ} \mathrm{C}$ for 5 days. The remaining portion was used for estimation of $\mathrm{C}$ Reactive Protein (CRP) and Total Leukocyte Count (TLC). All the samples were transported at room temperature as soon as possible to Microbiology Laboratory for further processing.

The inoculated BacT/ALERT PF Plus vials were placed in BacT/ALERT system. A positive result was indicated by an audible alarm and yellow illumination of the positive indicator lamp at the site of positive vial. On the computer instrument status displaying the station number was showed by flashing green in case of a positive vial. The bottles were incubated for five days before being reported as negative. A Gram stain and a subculture on blood agar, MacConkey agar and chocolate agar were performed from each presumptive positive vial. After incubation at $37^{\circ} \mathrm{C}$ the bacterial isolates were identified by gram staining, colony characteristics and a battery of biochemical tests following a standard protocol. ${ }^{8}$

\section{ANTIBIOTIC SUSCEPTIBILITY TESTS \\ Antibiogram}

Was done on Mueller-Hinton agar by the Kirby Bauer disc diffusion method as per Clinical Laboratory Standards Institute (CLSI) guidelines. ${ }^{9}$

Antibiotics were chosen for the study supplied by HiMedia Laboratories, Mumbai, according to their common use.

The strength of discs used and their zone size interpretative standards were according to guidelines by Clinical and Laboratory Standard Institute 2014 (CLSI) guidelines standards. ${ }^{9}$

The Antibiotic Panel used for Gram Positive Organisms: ${ }^{9}$ Amikacin $(30 \mu \mathrm{g}, \mathrm{Ak})$, Ampicillin sulbactam $(10 \mu \mathrm{g} / 10 \mu \mathrm{g}$, As), Azithromycin (15 $\mu \mathrm{g}, \mathrm{AZM})$, Cefoxitin (30 $\mu \mathrm{g}, \mathrm{Cx})$, Ciprofloxacin (5 $\mu \mathrm{g}$, CIP), Gentamicin (10 $\mu \mathrm{g}$, Gen), Linezolid $(30 \mu \mathrm{g}, \mathrm{Lz})$, Vancomycin $(30 \mu \mathrm{g}, \mathrm{VA})$.

The Antibiotic Panel used for Gram Negative Organisms: ${ }^{9}$

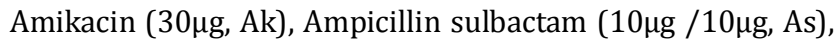
Amoxicillin clavulanate $(20 \mu \mathrm{g} / 10 \mu \mathrm{g}$, Amc), Ciprofloxacin (5

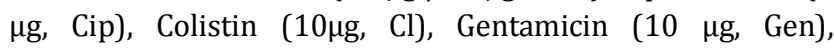
Ceftazidime (30 $\mu \mathrm{g}, \mathrm{Caz})$, Ceftazidime clavulanate $(30 / 10 \mu \mathrm{g}$, Cac), Imipenem (10 $\mu \mathrm{g}, \mathrm{Ipm})$, Meropenem (10 $\mu \mathrm{g}, \mathrm{Mrp})$, Piperacillin/ Tazobactam (100/10 $\mu$ g, Pit).

\section{RESULTS}

\section{Incidence of Sepsis}

In this study out of 100 neonates 38 (38\%) showed sepsis and $62(62 \%)$ showed no sepsis. Incidence of sepsis is shown in Graph 1. 


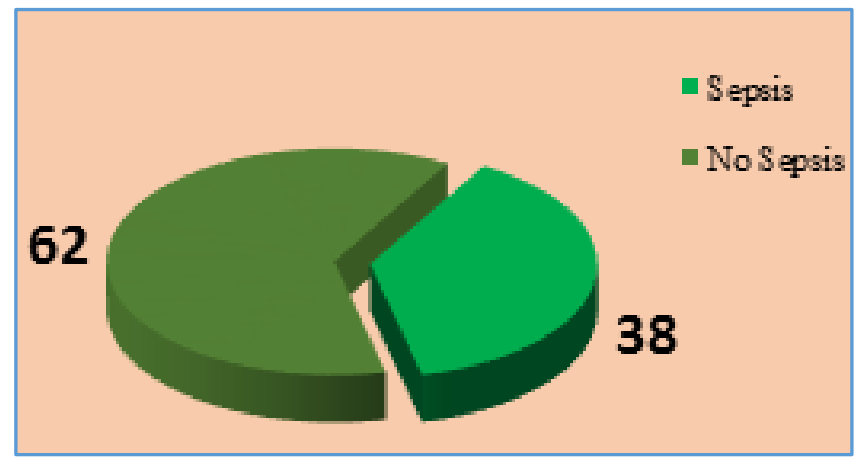

Graph 1: Incidence of Sepsis

Sex Wise Distribution

In this study $60 \%(23 / 38)$ males and $40 \%$ (15/38) females showed sepsis.

\begin{tabular}{|c|c|c|}
\hline Sex of the Child & Number & Percentage (\%) \\
\hline Male & 23 & 60 \\
\hline Female & 15 & 40 \\
\hline Total & $\mathbf{3 8}$ & $\mathbf{1 0 0}$ \\
\hline \multicolumn{3}{|c|}{ Table 1: Sex Wise Distribution among } \\
the Neonates having Septicaemia \\
\hline
\end{tabular}

Time of Isolation of Organisms

In the present study Early Onset Sepsis (EOS) constituted $58 \%(22 / 38)$ and Late Onset Sepsis (LOS) showed 42\% $(16 / 38)$. Early onset sepsis was more by $16 \%$ in the present study (Table 2).

\begin{tabular}{|c|c|c|}
\hline Time & Number & Percentage (\%) \\
\hline EOS $<72$ hrs. & 22 & 58 \\
\hline LOS $>72$ hrs. & 16 & 42 \\
\hline Total & $\mathbf{3 8}$ & $\mathbf{1 0 0}$ \\
\hline \multicolumn{2}{|c|}{ Table 2: Time of Isolation of Organisms } \\
\hline
\end{tabular}

Gestational Age of the Neonates having Septicaemia

In this study, 64\% (24/38) Preterm and 36\% (14/38) Term infants showed sepsis (Table 3).

\begin{tabular}{|c|c|c|}
\hline Gestational Age & Number & Percentage (\%) \\
\hline Preterm $<37$ weeks & 24 & 64 \\
\hline Term $\geq 37$ weeks & 14 & 36 \\
\hline Total & 38 & 100 \\
\hline
\end{tabular}

Distribution of Various Kinds of Microorganisms Isolated Out of 38 isolates the distribution of organisms was CONS $34 \%$ (13/38), Klebsiella pneumoniae 24\% (9/38), Acinetobacter spp. 24\% (9/38), Pseudomonas aeruginosa $7.8 \%$ (3/38), Enterococcus spp. 5\% (2/38), Serratia marcescens $2.6 \%$ (1/38), Candida spp. 2.6\% (1/38), (Table 4).

\begin{tabular}{|c|c|c|}
\hline Name of Isolate & Number & Percentage (\%) \\
\hline CONS & 13 & 34 \\
\hline Klebsiella pneumoniae & 9 & 24 \\
\hline Acinetobacter spp. & 9 & 24 \\
\hline Pseudomonas aeruginosa & 3 & 7.8 \\
\hline Enterococcus spp. & 2 & 5 \\
\hline
\end{tabular}

\begin{tabular}{|c|c|c|}
\hline Serratia marcescens & 1 & 2.6 \\
\hline Candida Spp. & 1 & 2.6 \\
\hline Total & 38 & 100 \\
\hline \multicolumn{3}{|c|}{$\begin{array}{c}\text { Table 4: Distribution of Various Kinds of Microorganisms } \\
\text { Isolated }\end{array}$} \\
\hline
\end{tabular}

Sensitivity Pattern of Antibiotics in Gram Negative Organisms

The in vitro antibiotic susceptibility pattern of Gram negative organisms showed very less sensitivity to Amoxicillin clavulanate (13.6\%), Amikacin (41\%), Ciprofloxacin (36.3\%) and Piperacillin tazobactam (41\%). Sensitivity to Gentamicin (54.6\%) and Ampicillin sulbactam (81.8\%) were good. They were $100 \%$ sensitive to Colistin, Imipenem and Meropenem as shown in Table 5.

\begin{tabular}{|c|c|c|c|c|c|}
\hline 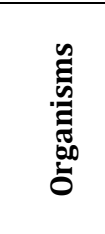 & 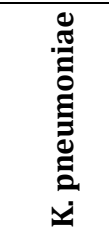 & 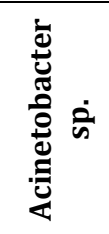 & م: 蒫 & 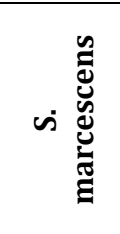 & 䒿 \\
\hline 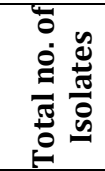 & 9 & 9 & 3 & 1 & 22 \\
\hline $\mathrm{AK}$ & $33 \%$ & $33 \%$ & $66 \%$ & $100 \%$ & $41 \%$ \\
\hline AS & $66 \%$ & $100 \%$ & $66 \%$ & $100 \%$ & $81.8 \%$ \\
\hline AMC & $22 \%$ & $0 \%$ & $33 \%$ & $0 \%$ & $13.6 \%$ \\
\hline CAZ & $33 \%$ & $0 \%$ & $100 \%$ & $100 \%$ & $31 \%$ \\
\hline CAC & $22 \%$ & $0 \%$ & $66 \%$ & $100 \%$ & $22.2 \%$ \\
\hline CIP & $44 \%$ & $0 \%$ & $100 \%$ & $100 \%$ & $36.3 \%$ \\
\hline CL & $100 \%$ & $100 \%$ & $100 \%$ & $100 \%$ & $100 \%$ \\
\hline GEN & $66 \%$ & $22 \%$ & $100 \%$ & $100 \%$ & $54.5 \%$ \\
\hline PIT & $44 \%$ & $22 \%$ & $66 \%$ & $100 \%$ & $41 \%$ \\
\hline IMP & $100 \%$ & $100 \%$ & $100 \%$ & $100 \%$ & $100 \%$ \\
\hline MRP & $100 \%$ & $100 \%$ & $100 \%$ & $100 \%$ & $100 \%$ \\
\hline Table & ensitiv & $\begin{array}{r}\text { Pattern } \\
\text { Org }\end{array}$ & $\begin{array}{l}\text { Antibio } \\
\text { issms }\end{array}$ & in Gra & Vegative \\
\hline
\end{tabular}

Sensitivity Pattern of Antibiotics in Gram Positive Organisms

The in vitro antibiotic susceptibility pattern of CONS showed moderate sensitivity to Amoxicillin clavulanate $(76 \%)$ and Ciprofloxacin (69\%). CONS showed $76 \%$ sensitivity to Cefoxitin. Enterococcus spp. isolates were resistance to Amikacin and Ciprofloxacin. They showed less sensitivity towards Gentamicin (50\%).

Gram positive organisms were $100 \%$ sensitive to Azithromycin, Linezolid and Vancomycin as shown in Table 6.

\begin{tabular}{|c|c|c|}
\hline Organisms & CONS & Enterococcus spp. \\
\hline Total no. of Isolates & 13 & 2 \\
\hline $\mathrm{AK}$ & $100 \%(13)$ & $0 \%(0)$ \\
\hline AS & $100 \%(13)$ & $0 \%(0)$ \\
\hline AMC & $76 \%(10)$ & \\
\hline AZM & $100 \%(13)$ & $100 \%(2)$ \\
\hline $\mathrm{CX}$ & $76 \%(10)$ & \\
\hline CIP & $69 \%(9)$ & $0 \%(0)$ \\
\hline GEN & $100 \%(13)$ & $50 \%(1)$ \\
\hline $\mathrm{LZ}$ & $100 \%(13)$ & $100 \%(2)$ \\
\hline VA & $100 \%(13)$ & $100 \%(2)$ \\
\hline \multicolumn{3}{|c|}{$\begin{array}{c}\text { Table 6: Sensitivity Pattern of Antibiotics in Gram Positive } \\
\text { Organisms }\end{array}$} \\
\hline
\end{tabular}




\section{DISCUSSION}

In present study, the incidence of septicaemia in neonates is $38 \%(38 / 100)$. Our study is comparable with study conducted by Chandra Madhur et al ${ }^{10}$ who also reported $37.63 \%$ culture positive cases.

The present study is correlated with study of Chandra Madhur et $\mathrm{al}^{10}$ in relation to time of isolation of organism and sex of the neonates. He reported that $56.32 \%$ belonged to early onset sepsis (0-3 days) and $43.68 \%$ belonged to late onset sepsis (4-28 days), he reported $62 \%$ were males and $38 \%$ were females.

Our study is comparable with study conducted by Omprakash Shukla et al ${ }^{11}$ who reported that $75 \%$ belonged to LBW ( $<2500 \mathrm{gms}$ ) and $25 \%$ were appropriate for gestational age ( $\geq 2500$ gms). He also reported preterm infants more than term infants were prone to septicaemia.

In the present study maximum number of isolates were gram negative organisms 22/38 (58\%), 15/38 (39.4\%) were gram positive organisms and $1 / 38(2.6 \%)$ was Candida species. This is similar to the study conducted by Omprakash Shukla et al. ${ }^{11} \mathrm{He}$ also reported that $69.7 \%$ were gram negative organisms and $30.23 \%$ gram positive organisms.

The commonest gram negative organisms in the present study were Klebsiella pneumoniae 9 (24\%), Acinetobacter spp. 9 (24\%) followed by Pseudomonas aeruginosa 3 (7.8\%), Serratia marcescens 1 (2.6\%). Gram positive organisms encountered in this study were CONS 13 (34\%), Enterococcus spp. (5\%) and Candida spp. 1 (2.6). These findings are close to results obtained by Tariq et $\mathrm{al}^{12}, \mathrm{Om}$ Prakash et $\mathrm{al}^{11}$ and Aduga Negusie et al. ${ }^{13}$

In the present study gram negative organisms showed less sensitivity to Amoxicillin clavulanate and Amikacin. Sensitivity result being Klebsiella pneumoniae 22\% and 33\%, Acinetobacter spp. $0 \%$ and $33 \%$, P. aeruginosa $33 \%$ and $66 \%$ and S. marcescens $0 \%$ and $100 \%$ respectively. Cephalosporins such as Ceftazidime and Ceftazidime clavulanate also showed less sensitivity towards gram negative organisms. Sensitivity results of Ceftazidime and Ceftazidime clavulanate being K. pneumoniae 33\%, 22\%, Acinetobacter spp. 0\%, 0\% respectively. P. aeruginosa $66 \%$ and $100 \%$ and Serratia marcescens $100 \%$ and $100 \%$ sensitive respectively.

Ciprofloxacin, Gentamicin and Piperacillin tazobactam also showed less sensitivity towards Klebsiella pneumonia and Acinetobacter spp., but Pseudomonas aeruginosa showed 66\% sensitivity towards Piperacillin tazobactam.

All the gram negative organisms showed $100 \%$ sensitivity towards Colistin, Imipenem and Meropenem. Our study is comparable with study conducted by Tariq Mahmud Tariq et al. 12

In the present study Coagulase Negative Staphylococci (CONS) showed moderate sensitivity (76\%) to Amoxicillin clavulanate and less sensitivity (69\%) to Ciprofloxacin. CONS showed $76 \%$ sensitivity to Cefoxitin, $100 \%$ sensitivity towards Azithromycin, Linezolid and Vancomycin.

In the present study, Enterococcus spp. showed 0\% sensitivity towards Amikacin and Ciprofloxacin. They showed $50 \%$ sensitivity towards Gentamicin and were $100 \%$ sensitive to Azithromycin, Linezolid and Vancomycin. Our study is comparable with study conducted by Mozhgan Shahian et al. ${ }^{14}$

\section{CONCLUSION}

We conclude that despite advances in diagnosis and treatment, bacterial sepsis remains a major cause of paediatric morbidity and mortality, particularly among neonates in developing countries. The management strategy therefore should focus on identification of risk factors; prompt laboratory screening of sepsis and early institution of empirical antibiotic treatment.

The isolation of microorganisms from blood is the gold standard method used to diagnose sepsis in the newborn infant. Neonatal septicaemia continues to pose a challenge to the paediatricians in making a definitive clinical diagnosis due to the subtle and nonspecific signs and symptoms; hence, laboratory diagnosis plays a major role. Definitive diagnosis rests on a positive blood culture to identify the pathogen and determine its antibiotic susceptibility pattern. We followed the strategy of BACTEC system while performing blood culture of the neonates. BACTEC is a sensitive method and lead to earlier detection of bacterial growth. Other important procedures to improve the sensitivity and specificity of blood cultures include proper skin disinfection before collection, culturing early in the septic episode, taking an appropriate volume of blood per culture. The diagnostic capabilities of blood culture systems have improved over the last decade with the advent of automated continuous blood culture monitoring systems. Although they can save time, subcultures are required for specific biochemical or other assays, ultimately needed for pathogen identification.

Blood culture is irreplaceable at the moment, since pure isolates are essential for antimicrobial drug susceptibility testing. The bacterial species responsible for sepsis vary depending upon the geographical location. In addition, knowledge of the likely causative organisms would contribute towards a more rational and appropriate use of antibiotics, thus minimizing the irrational use and emergence of multidrug resistant bacteria in neonatal units. The empirical regimen should be modified from time-to-time based on the antibiogram of isolates. Early identification of organisms causing neonatal sepsis and appropriate use of antibiotics also minimizes the morbidity and mortality of the neonates in neonatal intensive care units.

\section{REFERENCES}

1. Lawn JE, Cousens S, Zupan J. 4 million neonatal deaths: when? Why? Where? Lancet 2005;365(9462):891-900.

2. Stoll BJ. The global impact of neonatal infection. Clin Perinatology 1997;24(1):1-21.

3. Bang AT, Bang RA, Bactule SB, et al. Effect of homebased neonatal care and management of sepsis on neonatal mortality: field trial in rural India. Lancet 1999;354(9194):1955-61.

4. Report of National Neonatal Perinatal Database (National Neonatology Forum) 2002-2003.

5. Venkatesh M, Flores A, Luna RA, et al. Molecular microbiological methods in the diagnosis of neonatal sepsis. Expert Review of Anti-Infective Therapy 2010;8(9):1037-48.

6. Collee JG, Duguid JP, Faser AG, et al. Laboratory strategy in diagnosis of infective syndromes. Mackie and McCartney Practical medical microbiology. 14th edition. Churchill Livingstone 2012. 
7. Koneman EW, Allen SD, Janda WM, et al. Koneman's Color Atlas and Textbook of Diagnostic Microbiology. $6^{\text {th }}$ ed. Philadelphia: Lippincott Williams \& Wilkins, 2006.

8. Sham DF, Weissfeld AS, Forbes BA. Bailey \& Scott's Diagnostic Microbiology. $13^{\text {th }}$ edition. Elsevier 2014:870-1.

9. Clinical and Laboratory Standards Institute. Performance standards for antimicrobial disc susceptibility tests: approved standard. Clinical and Laboratory Standards Institute, Wayne, PA. 2014:M100S17.

10. Sharma CM, Agarwal RP. Neonatal sepsis: bacteria and their susceptibility pattern towards antibiotics in NICU. JCDR 2013;7(11):2511-3.
11. Shukla OS, Patel S, Vasava H. Clinical, epidemiological and microbiological profile of neonatal sepsis in NICU. Int J Res Med 2014;3(2):80-3.

12. Tariq TM. Bacteriologic profile and antibiogram of blood culture isolates from a children's hospital in Kabul. Journal of the College of Physicians and Surgeons Pakistan 2014;24(6):396-9.

13. Negussie A, Mulugeta G, Bedru A, et al. Bacteriological profile and antimicrobial susceptibility pattern of blood culture isolates among septicemia suspected children in selected hospitals addis ababa, ethiopia. Int J Bio Med Res 2015;6(1):4709-17.

14. Shahian M, Pishva N, Kalani M. Bacterial aetiology and antibiotic sensitivity patterns of EONS among neonates. IJMS 2010;35(4):293-8. 